\title{
Efeito do tratamento químico de sementes de arroz no controle da brusone nas folhas e na qualidade sanitária e fisiológica das sementes
}

\author{
Valacia L. da Silva Lobo \\ Embrapa Arroz e Feijão, Cx. Postal 179, 75375-000, Santo Antônio de Goiás, GO, Brasil, e-mail: valacia@cnpaf.embrapa.br
}

\begin{abstract}
RESUMO
Foram realizados dois experimentos de campo, em 2004 e 2005 com o objetivo de avaliar a eficiência de fungicidas no tratamento de sementes no controle de brusone nas folhas. Os mesmos fungicidas foram avaliados em laboratório, analisando-se o perfil fisiológico e sanitário das sementes tratadas. A análise conjunta dos experimentos mostrou diferenças significativas quanto aos valores da área abaixo da curva de progresso da doença (AACPD). Os tratamentos com pyroquilon e azoxystrobin foram os que propiciaram menor severidade de brusone nas folhas, apresentando as menores AACPD, enquanto o tratamento com carboxim + thiram não diferiu da testemunha no controle da doença. No entanto, este tratamento aumentou significativamente a germinação das sementes em relação à testemunha (22\%) e aos demais tratamentos. De maneira geral, todos os tratamentos foram eficientes em reduzir e/ou eliminar os fungos presentes nas sementes em laboratório, o carboxin + thiram e o tricyclazole foram os tratamentos que se destacaram.
\end{abstract}

Palavras-chave: Magnaporthe grisea, Oryza sativa, germinação, sanidade de sementes.

\begin{abstract}
Effects of chemical treatment of rice seeds on leaf blast control and physiological and sanitary quality of treated seeds

A field experiment was carried out in two successive years (2004 and 2005) in order to evaluate the effectiveness of the fungicides in rice seed treatment to control leaf blast. Another experiment was carried out in the laboratory to analyze the physiological and sanitary profile of treated seeds. The statistical analysis showed significant differences for the area under the disease progress curve (AUDPC). The pyroquilon and azoxystrobin treatments showed the least severity of leaf blast with the lowest AUDPC. Carboxim + thiram did not differ from the test to control leaf blast in the field, but this treatment increased seed germination (22\%) compared with the other treatments and with the untreated control. In general, all treatments were efficient in reducing and/or eliminating fungi in seeds, with best results from carboxin + thiram and tricyclazole.
\end{abstract}

Keywords: Magnaporthe grisea, Oryza sativa, blast disease, germination, seed health.

Dentre as doenças mais comuns e economicamente importantes, que afetam a cultura do arroz, está a brusone, causada pelo fungo Magnaporthe grisea (T.T. Hebert) M.E. Barr (anamorfo Pyricularia grisea Sacc). No Brasil, tanto a brusone nas panículas quanto nas folhas apresenta-se como um dos principais fatores que afetam a produtividade, tanto no sistema de cultivo de terras altas quanto no irrigado, impedindo que as cultivares expressem seu potencial produtivo. O cultivo extensivo da cultura do arroz, associado às condições climáticas favoráveis e às práticas culturais inadequadas, incluindo o uso de altas doses de nitrogênio e plantios adensados e/ou tardios, têm contribuído para aumentar a importância da brusone na região do Brasil Central. Apesar da doença ocorrer em todas as fases da cultura, sua ocorrência nas folhas entre os 30 e 50 dias após o plantio e nas panículas durante a formação dos grãos são as fases mais críticas, causando prejuízos significativos na produtividade. A severidade de brusone nas folhas é altamente influenciada pelo grau de resistência da cultivar, pela quantidade de inóculo inicial e pelas condições climáticas (Prabhu et al., 1999).

As sementes contaminadas, os restos culturais, e os esporos provenientes de lavouras infectadas constituem as principais fontes de inóculo primário do patógeno (Filippi \& Prabhu, 1997; Prabhu, 1988; Prabhu \& Morais, 1986). O uso de sementes sadias ou tratadas é importante para evitar a introdução de novos patótipos em novas áreas, principalmente devido ao fato deste patógeno apresentar variabilidade alta, um dos motivos pela baixa durabilidade da resistência das cultivares comerciais de arroz (Prabhu et al., 1999). Os métodos que reduzem o inóculo inicial incluem o uso de sementes sadias ou a erradicação do patógeno com produtos químicos ou outros tratamentos. $\mathrm{O}$ uso de fungicidas via aplicação aérea para o controle de brusone nas folhas é limitado, devido ao alto custo dos produtos e a baixa eficiência (Prabhu et al., 1999).

O tratamento de sementes é considerado como uma das práticas culturais de baixo custo, para proteger as 
culturas contra pragas e doenças em arroz de terras altas. Os fungicidas sistêmicos registrados especificamente para o tratamento de sementes contra brusone nas folhas incluem carboxim + thiram, thiabendazole, pyroquilon (Kimati et al., 1986). A eficiência desses produtos variam, bem como seus efeitos residuais. Apesar da importância da brusone no arroz, atualmente são poucos os fungicidas registrados para essa cultura, principalmente os de ação sistêmica. Os poucos produtos registrados, apresentam baixa atividade residual e alguns estão sendo utilizados há mais de vinte anos, outros já não apresentam a eficiência desejada.

A qualidade fisiológica da semente é avaliada por duas características fundamentais, a viabilidade e o vigor. A viabilidade, determinada pelo teste de germinação, procura avaliar a máxima germinação da semente. Enquanto, o vigor compreende um conjunto de características que determinam o potencial fisiológico das sementes, sendo influenciado pelas condições de ambiente e manejo durante as etapas de pré e póscolheita (Vieira \& Carvalho, 1994). Este experimento teve como objetivos avaliar a eficiência de fungicidas no tratamento químico de sementes no controle da brusone nas folhas e avaliar a eficiência dos mesmos na qualidade fisiológica e sanitária das sementes tratadas, em laboratório.

Foram conduzidos dois experimentos nos anos de 2004 e 2005, no campo experimental da Embrapa Arroz e Feijão, localizada no município de Santo Antônio de Goiás, GO. Os plantios foram feitos em 30 de janeiro de 2004 e 17 de janeiro de 2005. A alta pressão da doença foi induzida utilizando como disseminadora uma bordadura, constituída por quatro cultivares suscetíveis, 30 dias antes da semeadura do experimento, nas duas laterais do bloco, no sentido perpendicular às linhas. $\mathrm{O}$ delineamento experimental usado foi o de blocos ao acaso com cinco tratamentos e quatro repetições. As parcelas consistiram de 12 linhas de $1,0 \mathrm{~m}$ de comprimento, espaçadas de $0,10 \mathrm{~m} \mathrm{e}$ a densidade de semeadura foi de 100 sementes por metro linear da cultivar Primavera.

Os produtos e doses (g do i.a./100 kg de sementes) avaliados no tratamento de sementes foram: azoxystrobin (100 g); pyroquilon (400 g); tricyclazole $(225 \mathrm{~g})$ e carboxin + thiram $(60+60 \mathrm{~g})$. A testemunha foi tratada somente com o inseticida carbofuran $(20 \mathrm{~kg}$ do p.c./ha) como os demais tratamentos.

A avaliação da severidade da brusone nas folhas iniciou-se aos 30 dias após a semeadura, nas três folhas do perfilho principal, em 20 plantas por parcela tomadas ao acaso, nas quatro linhas centrais, utilizando-se a escala de 10 graus $(0 ; 0,5 ; 1 ; 2 ; 4 ; 8 ; 32 ; 64 ; 82$ e $100 \%$ de área foliar afetada), de acordo com Notteghem (1981). Foram feitas cinco avaliações em intervalos de sete dias, para o cálculo da severidade de acordo com a área abaixo da curva do progresso da doença (AACPD).
As sementes, utilizadas no ensaio de 2005, foram analisadas quanto a sanidade e a germinação. Para a análise da germinação foram utilizadas, para cada tratamento, quatro repetições de 50 sementes colocadas em papel germitest, umedecido com água destilada. A contagem das plântulas normais foi feita após sete e dez dias. A qualidade sanitária das sementes foi analisada utilizando-se oito repetições de 25 sementes para cada tratamento. O método usado foi o de papel-de-filtro (blotter). As sementes foram incubadas por um período de sete dias, a $20 \pm 2{ }^{\circ} \mathrm{C}$, em regime intermitente de $12 \mathrm{~h}$ de luz/12 h de escuro. Após o período de incubação foi feita a identificação dos fungos por semente e sua incidência expressa em porcentagem. As análises de germinação e sanidade foram feitas de acordo com as Regras para Análise de Sementes (Brasil, 1992). Os dados foram submetidos à análise de variância utilizando o programa estatístico SAS e para a comparação das médias foi utilizado o teste de Tukey ao nível de 5\% de probabilidade.

A análise conjunta dos experimentos de campo mostrou diferenças significativas nos valores da AACPD, indicando diferenças entre os tratamentos (Figuras 1 e 2). A menor severidade de brusone foi observada nas parcelas tratadas com os fungicidas azoxystrobin e pyroquilon, os quais não diferiram entre si. O fungicida tricyclazole apresentou resultado intermediário enquanto carboxim + thiram não diferiu da testemunha (Figura 2).

$\mathrm{Na}$ análise de germinação, carboxim + thiram (87\%) diferiu significativamente dos demais tratamentos, apresentando um acréscimo do poder germinativo de $22,54 \%$ em relação à testemunha $(71 \%)$. Quando se utilizou os tratamentos azoxystrobin (58\%) e pyroquilon (59\%) observou-se uma redução da germinação da ordem de $17 \%$ com o uso de azoxystrobin e $16 \%$ com
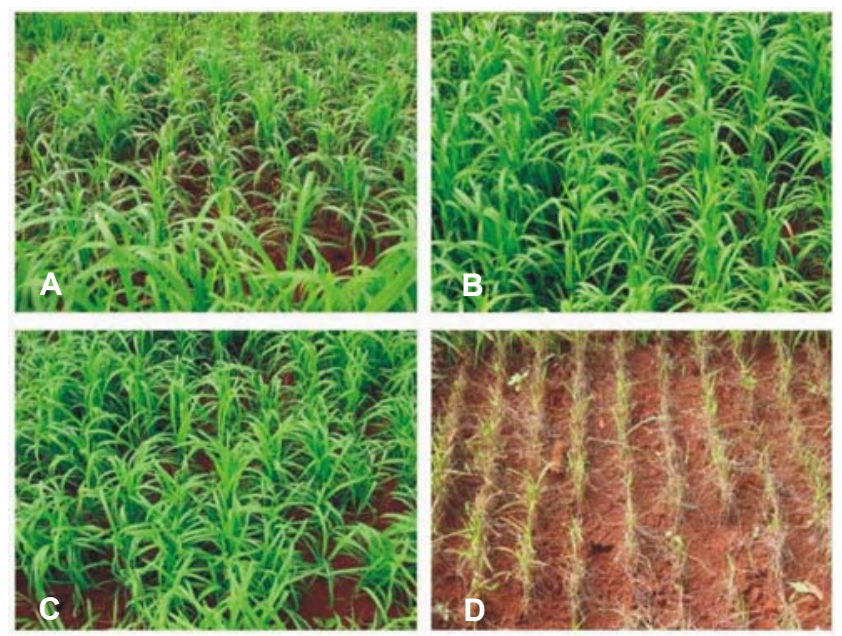

FIG. 1 - Efeito do tratamento químico de sementes de arroz no controle da brusone nas folhas. A. Tricyclazole; B. Axoxystrobin; C. Fongorene; D. Parcela sem tratamento 


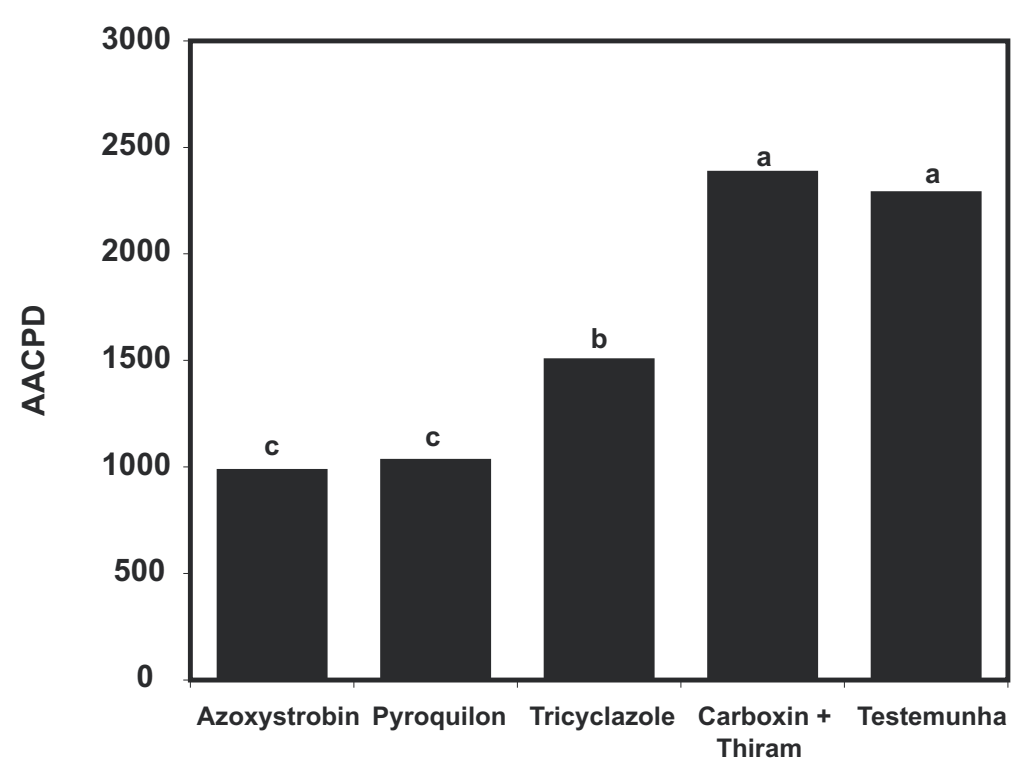

Tratamentos
FIG. 2 - Eficiência do tratamento químico de sementes no controle de brusone nas folhas em arroz. pyroquilon, em relação à testemunha, apesar destes tratamentos não diferirem desta. O tricyclazole apresentou a mesma porcentagem de germinação que a testemunha. Para análise de sanidade realizada em laboratório, de maneira geral, todos os tratamentos foram eficientes em eliminar ou reduzir os fungos presentes nas sementes de arroz, destacando-se o tratamento carboxim + thiram e tricyclazole (Tabela 1).

Em ensaios anteriores e mesmo em lavouras comerciais, carboxin + thiram mostrou-se eficiente em controlar a brusone no campo, inclusive melhorando a germinação das sementes contaminadas (Silva-Lobo, 2005), porém na dose utilizada neste experimento, sob condições de alta pressão de inóculo e condições ambientais favoráveis, o mesmo não ofereceu proteção contra a doença no campo, apesar de melhorar significativamente a germinação das sementes infectadas e eliminar ou reduzir outros fungos presentes nas sementes de arroz, em ensaio de laboratório. É importante salientar que esses fungos fazem parte do complexo de patógenos responsáveis pela doença mancha de grãos, que é a segunda em importância para a cultura do arroz, principalmente Drechslera oryzae, Phoma sp. e Microdochium oryzae, este último agente causal da escaldadura do arroz. A redução e/ou eliminação dos fungos das sementes explica a melhora da germinação das sementes, visto que carboxim + thiram ao lado do tricyclazole foi o mais eficiente em melhorar a sanidade das sementes (Tabela 1; Figura 3).

$\mathrm{O}$ fungicida pyroquilon foi um dos tratamentos que proporcionou o melhor controle de brusone nas folhas. Sua eficiência vem sendo comprovada ao longo dos anos em que o produto vem sendo utilizado (Filippi \& Prabhu, 1997; Teixeira et al., 1997; Silva et al., 2002). Este produto foi retirado, recentemente, do mercado e no momento não existe registro de outro produto equivalente para o tratamento de sementes, que visa o controle de brusone nas folhas.

No Brasil, os fungicidas tricyclazole e azoxystrobin são recomendados e registrados para o controle de brusone via aplicação foliar, mas não para tratamento de sementes; no entanto, nas últimas safras tem se observado um grande número de produtores utilizando esses produtos no tratamento de sementes. Estes fungicidas, quando aplicados no tratamento de sementes, apresentaram um bom controle da doença nas folhas e não se verificou fitotoxidez ou quaisquer outros problemas na utilização dos mesmos.

Em relação ao perfil fisiológico das sementes, Prabhu \& Vieira (1989) observaram que sementes de arroz, tratadas com carboxim + thiram, apresentaram aumento na germinação e também melhor sanidade das plântulas. Sementes de sorgo tratadas com este fungicida e armazenadas em câmara fria apresentaram melhor conservação do vigor quando comparadas àquelas armazenadas sem receber o tratamento (Netto et al., 1997). O mesmo comportamento foi observado em sementes de algodão (Faria et al., 2003).

Os resultados evidenciam a necessidade de desenvolvimento e registro de novas moléculas para o controle de brusone nas folhas via tratamento de sementes. Apesar do baixo desempenho de carboxim + thiram no controle da brusone no campo, sob alta 
Efeito do tratamento químico da semente de arroz no controle da brusone...

TABELA 1 - Efeito do tratamento químico de sementes de arroz na qualidade fitossanitária, avaliada pelo método do papel filtro

\begin{tabular}{|c|c|c|c|c|c|c|}
\hline Tratamento & Dose $^{a}$ & $\begin{array}{c}\text { Drechslera } \\
\text { oryzae }\end{array}$ & $\begin{array}{c}\text { Fusarium } \\
\text { moniliforme }\end{array}$ & $\begin{array}{c}\text { Microdochium } \\
\text { oryzae }\end{array}$ & $\begin{array}{l}\text { Phoma } \\
\text { sp. }\end{array}$ & $\begin{array}{c}\text { Pyricularia } \\
\text { grisea }\end{array}$ \\
\hline Testemunha & - & $21^{\mathrm{b}}$ & 61,5 & 22,5 & 66,5 & 4,67 \\
\hline Carboxim + Thiram & $60+60$ & 0 & 1 & 0 & 0,5 & 0 \\
\hline Tricyclazole & 225 & 3 & 0 & 0 & 0 & 0 \\
\hline
\end{tabular}

a g do i.a./100 Kg de sementes.

${ }^{\mathrm{b}}$ Porcentagem de sementes infectadas pelo fungo.

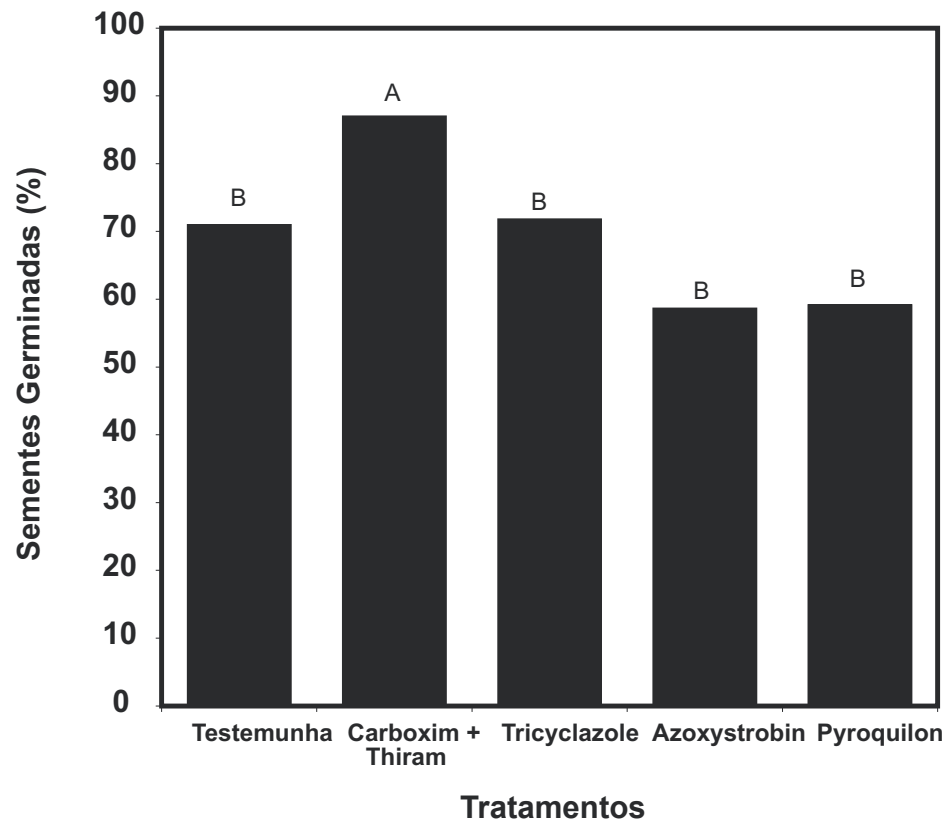

FIG 3 - Efeito do tratamento químico de sementes de arroz, com fungicidas, sobre a germinação. pressão da doença e condições climáticas favoráveis, este fungicida apresentou um alto desempenho, melhorando a qualidade fisiológica e sanitária das sementes, assim como o tricyclazole, quando avaliados em laboratório.

\section{REFERÊNCIAS BIBLIOGRÁFICAS}

Brasil (1992) Ministério da Agricultura e Reforma Agrária. Regras para análise de sementes. Brasília DF. SNDA/DNDV/CLAV.

Faria AYK, Albuquerque MCF, Cassetari Neto D (2003) Qualidade fisiológica de sementes de algodoeiro submetidas a tratamentos químicos e biológico. Revista Brasileira de Sementes 25:121-127.
Filippi MC, Prabhu AS (1997) Integrated effect of host plant resistance and fungicidal seed treatment on rice blast control in Brazil. Plant Disease 81:351-355.

Kimati H, Soave J, Eskes AB, Kurozawa C, Brignani Neto F, Fernandes NG (1986) Guia de fungicidas agrícolas. Piracicaba SP. Grupo Paulista de Fitopatologia.

Netto DAM, Borba CS, Oliveira AC, Azevedo JT, Andrade RV, Andreoli C (1997) Qualidade fisiológica de sementes de sorgo após armazenamento. Revista Brasileira de Sementes 19:342-348.

Notteghem JL (1981) Cooperative experiment on horizontal resistance to rice blast. In: Blast and upland rice: report and recommendation from the meeting for international collaboration in upland rive improvement. Los Baños. International Rice Research Institute, pp. 43-51.

Prabhu AS (1988) Controle das principais doenças de arroz de 
V.L.S. Lobo

sequeiro. Informe Agropecuário 14. pp. 58-63.

Prabhu AS, Morais OP (1986) Blast disease management in upland rice in Brazil. Proceedings, International Upland Rice Conference, 1985, Jakarta, Indonesia. Progress in upland rice research. Manila, pp. 382-394.

Prabhu AS, Filippi MC, Ribeiro AS (1999) Doenças e seu controle. In: Vieira NRA, Santos AB, Sant'Ana EP (Eds.) A cultura do arroz no Brasil. Santo Antônio de Goiás GO. Embrapa Arroz e Feijão. pp. 262-307.

Prabhu AS, Vieira NRA (1989) Sementes de arroz infectadas por Drechslera oryzae: germinação, transmissão e controle. Goiânia GO. Embrapa Arroz e Feijão.

Silva GB, Cezar MC, Filippi MC, Prabhu AS (2002) Resposta de cultivares melhoradas de arroz ao tratamento de sementes com pyroquilon no controle da brusone nas folhas. Summa Phytopathologica 28:289-291.

Silva-Lobo VL (2005) Tratamento químico de sementes de arroz no controle da brusone nas folhas. Fitopatologia Brasileira 30:159.

Soave J, Moraes SA (1987) Medidas de controle das doenças transmitidas por sementes. In: Soave J, Wetzel MMVS (Eds.) Patologia de sementes. Campinas SP. Fundação Cargill. pp. 192259.

Teixeira EL, Prabhu AS, Filippi MC (1997) Eficiência relativa dos fungicidas sistêmicos, no tratamento de sementes para o controle da brusone nas folhas de arroz Revista Brasileira de Sementes 19:180-185.

Vieira RD, Carvalho NM (1994) Testes de vigor em sementes. Jaboticabal SP. Fundação de Apoio a Pesquisa, Ensino e Extensão. 